\title{
Modified DAG Location for Delaunay Triangulation
}

\author{
Ivana Kolingerová ${ }^{1}$ \\ Centre of Computer Graphics and Data Visualization, Department of Computer Science and \\ Engineering, University of West Bohemia, Plzeň \\ kolinger@kiv.zcu.cz, http://iason.zcu.cz/ kolinger
}

\begin{abstract}
The paper describes a modification of DAG-based location for incremental insertion Delaunay triangulation algorithm in $E^{2}$ and $E^{3}$. Instead of the whole simplices, only their separating faces are stored and tested. The proposed technique allows full reuse of results obtained on previous DAG levels. This enables to reduce time for point location and memory requirements. Asymptotic memory and time complexity is not changed.
\end{abstract}

\section{Introduction}

Incremental-insertion-based algorithms do not belong to the fastest but they are easy to understand, on-line, their implementation is usually quite simple and it is a bit easier to make them robust than other types of algorithms, e.g. divide-and-conquerbased. The complexity of incremental insertion algorithms is given as $n^{*} C 1$ where $C 1$ is complexity needed to process one element and $n$ is the number of elements. Therefore, they typically cannot achieve better complexity than $O\left(n^{2}\right)$ in the worst case and, with the help of auxiliary data structures, $O(n \log n)$ or even $O(n)$ in expected case for most type of input data distribution.

These general features fully pay also for incremental insertion algorithm for constructing Delaunay triangulation in $E^{2}$ and $E^{3}$. The algorithm is quite simple; to be effective, it is combined with such a data structure which enables efficient location of newly inserted points in the already finished part of triangulation; usually some kind of tree or uniform space subdivision. With such a data structure, this algorithm has $O\left(n^{2}\right)$ worst case and $O(n \log n)$ up to $O(n)$ expected case time complexity in $E^{2}$ and $O\left(n^{3}\right)$ worst case and $O(n \log n)$ up to $O(n)$ expected case time complexity in $E^{3}$. $\left(O\left(n^{3}\right)\right.$ bound for $E^{3}$ can appear if the resulting triangulation has $O\left(n^{2}\right)$ simplices - it is possible but very rare, see $[5,6])$.

One of the oldest and most often used data structure is a DAG, directed acyclic graph. One node of DAG contains one simplex. Using DAG and a randomization of the input data, optimal $O(\log n)$ location time per inserted point can be achieved in the expected case. Even better, its behaviour for various input data distribution is nearly identical. The most important disadvantage of DAG are big memory requirements although in $E^{2}$ asymptotically linear, the memory for DAG reaches up to $9 n$ according

${ }^{1}$ This work was supported by the Ministry of Education of The Czech Republic - project MSM 235200005. 
to our measurements ( $468 n$ bytes in our implementation). For $E^{3}$, the memory is asymptotically quadratic because number of tetrahedra in a triangulation in $E^{3}$ can be quadratic; in practice, we measured $54 n$ ( $3672 n$ bytes in our implementation) as an upper limit for various input distributions and real data.

In this paper, we will show how time for point location and memory requirements of DAG can be reduced if, instead of the whole simplices, only separating faces are stored in DAG and tested, thus reducing redundancy in the hierarchical point-insimplex tests. The proposed technique has some similarity to miniDAG location used for regular triangulation in $E^{3}$ in [7]; however, it was developed independently. In [12], we gave a slightly different version of this technique in $E^{2}$ as a practical algorithm for computer graphics and GIS applications. This paper shows further development of this technique and its $E^{3}$ generalization.

Section 2 gives all necessary definitions, briefly describes the fundamental algorithm and surveys previous work on fast location for incremental Delaunay insertion. Section 3 provides details about DAG for Delaunay triangulation. Section 4 explains the newly proposed modification and section 5 handling of singularities. Section 6 gives theoretical and experimental results. Section 7 concludes the paper.

\section{Background}

Given a point set $P$ in $E^{d}$, a $k$-simplex, $k \leq d$, is a convex combination of $k+1$ affinely independent points in $P$, called vertices of the simplex. An $s$-face of a simplex is the convex combination of a subset of $s+1$ vertices of the simplex. By 'face' we will mean $d$-1-face. [2]

A triangulation $T(P)$ of a set of points $P$ in $E^{\mathrm{d}}$ is the set of $d$-simplices such that:

1. a point $p$ in $E^{d}$ is a vertex of a simplex in $T(P)$ if $p \in P$

2. the intersection of two simplices in $T(P)$ is either empty or a common vertex or a common face

3. the set $T(P)$ is maximal; it is not possible to add any simplex without violating the previous rules.

A triangulation $T(P)$ in $E^{d}$ is a Delanauy triangulation if the circumsphere of each $d$-simplex does not contain any point of the set $P$.

We suppose that the orientation of simplex faces is positive. To decide whether a point is inside a simplex, we use the orientation predicate, defined for $E^{d}$ over $d+1$ points by the sign of $d$-dimensional determinant. For $E^{2}$ and $E^{3}$, these predicates can be as follows:

$$
\left.E^{2}: \quad \operatorname{Right}(a, b, c)=\operatorname{Sgn}\left(\begin{array}{ll}
b y-a y & c y-b y \\
b x-a x & c x-b x
\end{array}\right)\right)
$$




$$
E^{3}: \operatorname{Right} 3 \mathrm{D}(a, b, c, d):=\operatorname{Sgn}\left(\left|\begin{array}{lll}
d x-a x & d y-a y & d z-a z \\
d x-b x & d y-b y & d z-b z \\
d x-c x & d y-c y & d z-c z
\end{array}\right|\right)
$$

Let's point out that for positive orientation, these predicates return minus if $c$ is to the left of $a b$ in $E^{2}$ and $d$ to the left of $a b c$, it means, if it is inside the simplex whose face $a b$ or $a b c$ is. The whole point-in-simplex test than calls the Right or Right3D function for the faces of the simplex. If the tested point is outside, we can stop the orientation test with the first positive sign; it saves a half of the tests in average. It means that 1.5 tests for a triangle and 2 tests for a tetrahedron are needed in average. If the point is inside, we have to test all faces. Singularities are indicated by at least one zero from orientation tests. Implementation of these predicates has to be robust enough to handle such cases, see e.g. [7].

Delaunay triangulation algorithm using DAG can be found for $E^{2}$ in $[1,9]$ and for $E^{3}$ in $[10,11,7]$. It achieves $O(\log n)$ per point location. We will not give a detailed explanation here because the algorithm is widely known. Brief description is as follows. At the beginning, all points of the input set $P$ are emebeded into one big auxiliary simplex. Then points are inserted one at a time. For each inserted point, the simplex containing it has to be found. This simplex is then subdivided into several simplices; three in $E^{2}$ and four in $E^{3}$. Then Delaunay triangulation has to be restored. It is done by tests of new simplices: The outer face of the new simplex is tested and if the empty circumsphere property is not satisfied, simplices sharing this face are swapped, i.e., changed to the other possible configuration. This swap is easy in $E^{2}$ because instead of two old, two new triangles are created, and difficult in $E^{3}$ where three kinds of swaps are possible: either two tetrahedra are replaced by three, or vice versa; the last possibility is a special swap of such two or four tetrahedra which together form a pyramid or a double pyramid with four vertices in one plane. (More details about swappable and non-swappable configurations in $E^{3}$ can be found in [10, 11] and are not subject of this paper.) All simplices are stored as nodes of DAG. When all points of $P$ have been inserted, all simplices containing the auxiliary points (which do not belong to $P$ ) are deleted and the final triangulation is extracted from DAG leaves.

Due to popularity of the incremental insertion algorithm, various point location techniques and data structures appeared, especially in $E^{2}$. Simple and popular is a strategy of walking, where the triangle containing the inserted point is found by visiting triangles between the inserted point and some starting point. This technique was for triangulations demonstrated in [8], achieving $O\left(n^{1 / 2}\right)$ per point location in $E^{2}$. Further improvement uses the idea of 'better starting point' estimate by random sampling $m$ middle centres of triangulation edges, $m=n^{1 / 3}$, and taking the point closest to the located point as a start of walking, see [14]. For randomly distributed points, $O\left(n^{1 / 3}\right)$ in $E^{2}$ and $O\left(n^{1 / 4}\right)$ in $E^{3}$ for one point location is achieved. Comparison of several walking strategies in Delaunay triangulation in $E^{2}$ and $E^{3}$ is given in [4].

[16] is a combination of walking, regular grid and presorting of points so that points neighbouring in the data structure are geometrically close. Tests show approximately $O\left(n^{1.1}\right)$ time per point location in $E^{2}$. 
[13] suggests a data structure with $O(\log n)$ levels, each level containing a random sample of triangles from the lower level of the data structure. At each level, Delaunay triangulation of the sample of points is computed and overlapping triangles at different levels are linked to ease a point-in-triangle location. One point location needs $O\left(\log ^{2} n\right)$ time. Simplification and improvement of this data structure can be found in [3] where $O(\log n)$ per point is achieved in $E^{2}$.

Another way to speed up point location is to use bucketing or quadtree data structures, see, e.g., [15, 18, 20]. Generally, this kind of data structure is more sensitive to input data distribution, although for more or less uniformly distributed points can be very fast.

The incremental insertion algorithm has also been implemented for non-euclidean metrics [19] and for constrained triangulation [18].

\section{Details about DAG for Delaunay triangulation}

DAG in the incremental insertion for Delaunay triangulation is a hierarchy in which a history of the triangulation is stored. For location, it is necessary to know links from parent nodes to sons to be able to traverse the DAG down to the leaves. Links in the opposite direction are not necessary. See example of a part of a triangulation history in a DAG in Fig. 1.

In $E^{2}$, a DAG contains three types of inner nodes:

1. nodes with three sons obtained by point insertion - three new triangles are made instead of one old,

2. nodes with two sons obtained by an insertion a point incident to an existing triangulation edge - four new triangles are made instead of two old, and

3. nodes with two sons obtained by an edge swap between two triangles - two new triangles are made instead of two old.

In $E^{3}$, there are these six types of inner nodes:

1. nodes with four sons obtained by point insertion - four new tetrahedra are made instead of one old,

2. nodes with three sons obtained by an insertion a point lying in a tetrahedron face six new tetrahedra are made instead of two old,

3. nodes with two sons obtained by an insertion of a point incident with an edge of the triangulation - each tetrahedron sharing this edge is divided into two tetrahedra; the total number of tetrahedra sharing an edge is usually a small constant but can be as high as $O(n)$,

4. nodes with three sons obtained by a face swap S23 - two tetrahedra are removed and replaced by three,

5. nodes with two sons obtained by a face swap S32 - three tetrahedra are removed and replaced by two,

6. nodes with two sons obtained by a face swap S44 or S22 - four or two new tetrahedra appear instead of the same number of old ones.

In any moment, leaves contain the current triangulation. 


\section{The proposed modification}

We will suppose that the orientation test returns plus or minus, never zero; handling of singularities will be explained in section 5. We will call Test $i j$ the orientation test Right() (in $E^{2}$ ) or Righ3D() (in $E^{3}$ ) of the face separating a simplex $i$ from simplex $j$, both simplices are sons of the same node.
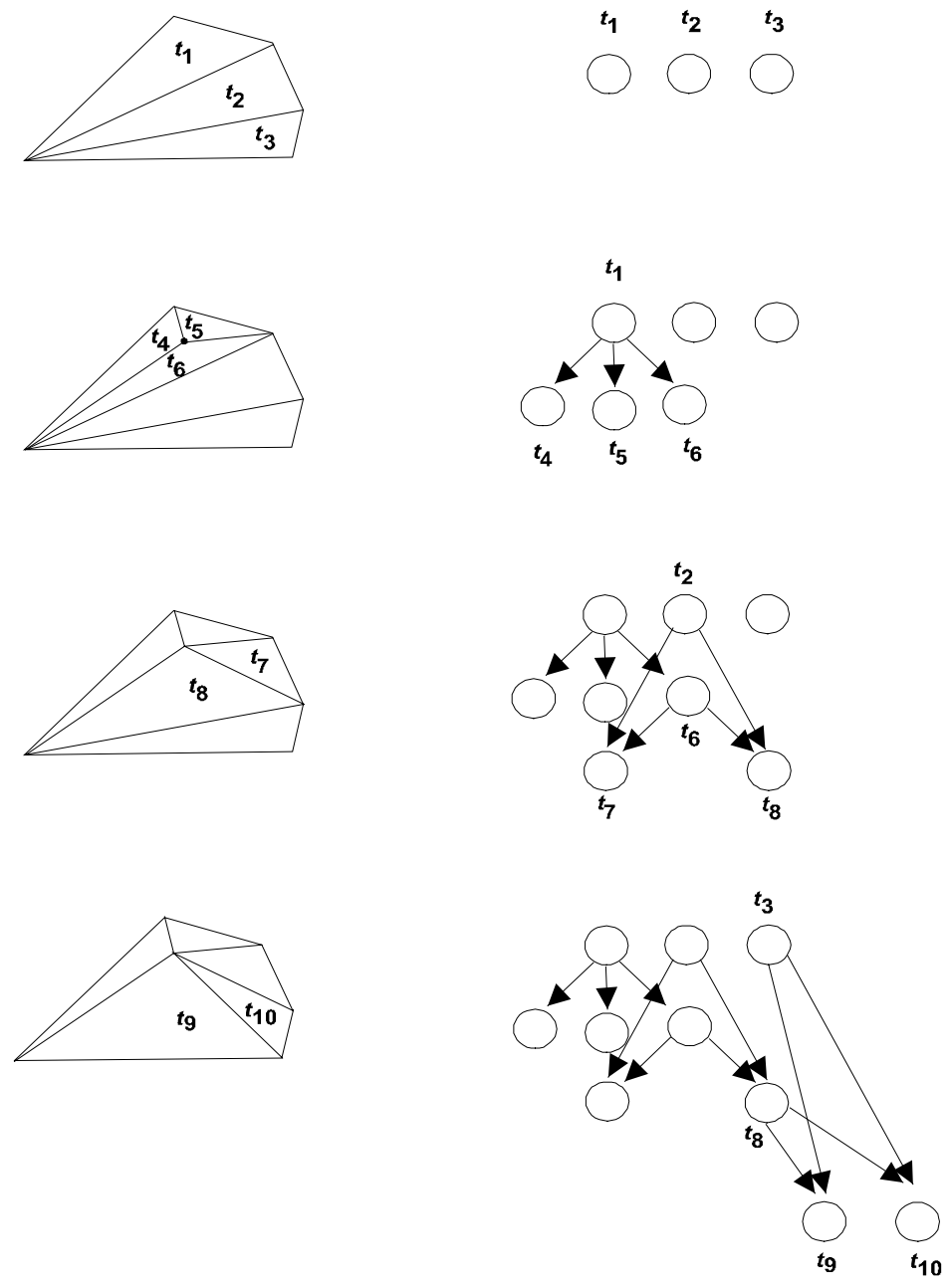

Fig. 1. Example of a part of a triangulation history in a DAG

For better readability, we will explain the proposed modification for $E^{2}$ case and then we will state differences for $E^{3}$.

Let's have such an inner node $N_{\mathrm{i}}$ of DAG which contains a triangle $t_{\mathrm{i}}$ that was found to contain the inserted point $q$. Let $t_{\mathrm{i}}$ have three sons $t_{0}, t_{1}, t_{2}$. All sons are tested by point-in-triangle tests, it means 9 orientation tests in the worst case and 4.5 in the 
average case. The first simple improvement that can be made is not to test the shared edges twice (once for each incident triangle), thus saving up to three tests for three sons. But the idea can be derived a bit further.

If we are testing the query point $q$ against triangles $t_{0}, t_{1}, t_{2}$, we already know that $q$ lies inside $t_{\mathrm{i}}$ because it has been tested in the previous step of the search traversal the outer boundary of $t_{\mathrm{i}}$ is in this case identical with outer boundary of its sons and repeating of its tests is useless. Tests of the edges separating $t_{0}, t_{1}, t_{2}$ are enough, see Fig.2a. Of these three edges, just two tests are necessary to distinguish all cases, see Table $1-$ e.g., the test Test 01 of the edge separating $t_{0}$ and $t_{1}$ distinguishes between $t_{0}$ and $t_{1}$ but does not help for $t_{2}$ because $t_{2}$ can have both signs, therefore, one more test has to be done after Test_01. The resulting sequence of tests is in Fig.3.
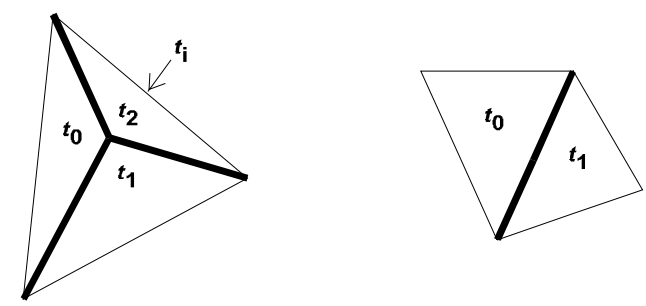

Fig. 2a. Separating edges in a node with three sons. 2b. In a node with two sons

Table 1. Which tests are necessary to distinguish whether a point lies in $t_{0}, t_{1}$ or $t_{2}$ if $t_{0}, t_{1}, t_{2}$ are sons of one triangle. Test $i j$ is an orientation test by the separating edge between $t_{\mathrm{i}}$ and $t_{\mathrm{j}}$. The symbol ' $\bullet$ ' means that both plus and minus is a possible result for this triangle.

\begin{tabular}{cccc}
\hline & Test_01 & Test_12 & Test_20 \\
\hline$t_{0}$ & - & $\bullet$ & + \\
$t_{1}$ & + & - & $\bullet$ \\
$t_{2}$ & $\bullet$ & + & - \\
\hline
\end{tabular}

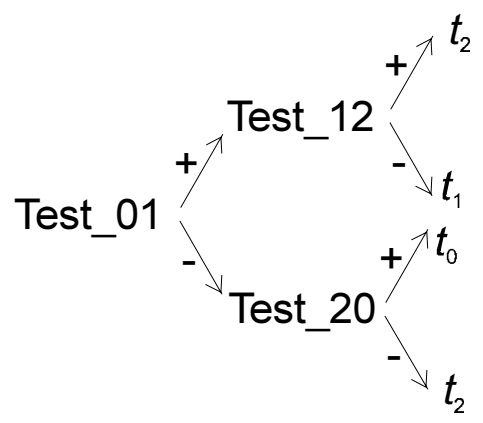

Fig. 3. A sequence of tests for 3 sons gives a structure of the corresponding algorithm

If $N_{\mathrm{i}}$ had two sons, the two triangles $t_{0}$ and $t_{1}$ would give together 6 and 3 orientation tests in the worst and average case, respectively. However, one test is 
enough to distinguish between $t_{0}$ and $t_{1}$, see Fig. $2 \mathrm{~b}$, even in case that they originated from a swap where new triangles are not covering the area of just one triangle but two, see $t_{2}, t_{7}, t_{8}$ or $t_{6}, t_{7}, t_{8}$ in Fig. 1 for better understanding. The sequence of tests is trivial here, just Test_01.

In the average case we save 2.5 tests per triangle with 3 sons ( 2 instead of 4.5 tests) and 2 tests per triangle with 2 sons ( 1 instead of 3 tests). In the worst case we would save 7 tests for 3 sons and 5 tests for 2 sons but the average case is more probable (recall Section 2).

Now to the $E^{3}$ case. For 2 and 3 sons, the sequence of tests is the same as in $E^{2}$. For 4 sons, there exist 6 separating faces - we need to handle 6 mutual combinations of tetrahedra. Table 2 shows which test distinguishes between which pair of tetrahedra. Of these 6 tests, we need 3 to find unambiguously which tetrahedron contains the point, see Fig.4.

Table 2: Which tests are necessary to distinguish whether a point lies in $t_{0}, t_{1}, t_{2}$ or $t_{3}$ if $t_{0}, t_{1}, t_{2}$, $t_{3}$ are sons of one tetrahedron. Test $i j$ is the orientation test by the separating face between $t_{i}$ and $t_{\mathrm{j}}$. The symbol ' $\bullet$ ' means that both plus and minus is a possible result for this tetrahedron.

\begin{tabular}{ccccccc}
\hline & Test_01 & Test_02 & Test_03 & Test_12 & Test_13 & Test_23 \\
\hline$t_{0}$ & - & - & - & $\bullet$ & $\bullet$ & $\bullet$ \\
$t_{1}$ & + & $\bullet$ & $\bullet$ & - & - & $\bullet$ \\
$t_{2}$ & $\bullet$ & + & $\bullet$ & + & $\bullet$ & - \\
$t_{3}$ & $\bullet$ & $\bullet$ & + & $\bullet$ & + & + \\
\hline
\end{tabular}

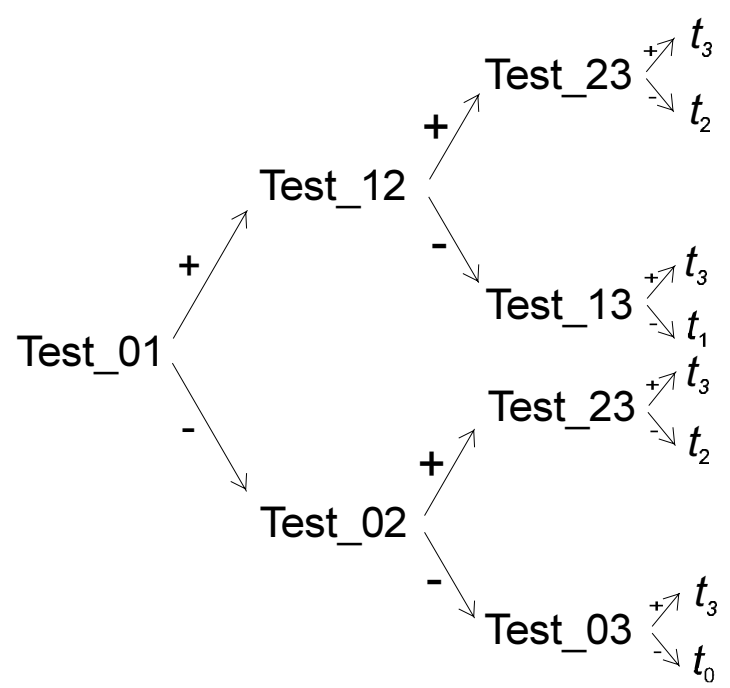

Fig. 4. A sequence of tests for 4 sons gives a structure of the corresponding algorithm

The proposed technique can be also used to reduce memory requirements of the DAG. It is not necessary to store all vertices of simplices if the simplices are not in the leaves; for DAG traversal, we need only part of vertices. Therefore, when a list becomes an inner node, we can deallocate part of its information. We described 
results of experiments with memory deallocation in [12] - in $E^{2}$, in our implementation about $28 \%$ memory savings were achieved, memory deallocation prolonged the computation $9 \%$ in average.

Before we will show estimated and measured savings, let us explain how to handle singularities - the inserted point lies on some face.

\section{Singularities}

It is necessary to recognize whether the tested point lies on some face to avoid creating simplices with empty volume. The orientation predicates return zero in such a case. However, if we are not in a leaf simplex, incidence of the tested point with some face is not substantial because the face may have been swapped and may not exist any more in the current triangulation. Therefore, it is useless to handle singularities earlier than at leaf level. For simplicity, we test singular cases by one extra call to point-in-simplex test, with full control of singular cases, at the end of DAG traversal, on leaf level. The amount of extra orientation tests caused by this suboptimal but simple solution is very small in comparison with the total number of tests (5\% for $n=1,000$ down to $2.3 \%$ for $n=1,000,000$ in $E^{2}$ and $3.8 \%$ for $n=1,000$ down to $1.8 \%$ for $n=240,000$ in $E^{3}$ ).

\section{Results}

The implementation was done in Delphi 5 on Windows NT and experiments were run on Dell Precision 410 (Pentium III, $700 \mathrm{MHz}, 1 \mathrm{~GB}$ RAM). Running times are averages for 5 point sets. Tests were done for uniform distribution, cluster distribution (several uniformly distributed points are centres of normal distribution, see [17] and for real data sets (GIS applications, surface models). As results for various types of input data were nearly the same (for cluster and real data even slightly better), we present in detail results for uniform data only. The tested data sets were up to $1,000,000$ points for $E^{2}$ and up to 240,000 points for $E^{3}$.

Measurements acknowledge $O(n \log n)$ time complexity of the DAG-based algorithm in the expected case both in $E^{2}$ and in $E^{3}$. Table 3 shows number of tested edges in $E^{2}$ per one point in the original and the improved method, their rate according to the experiments and according to a theoretical estimate. (Estimates were done according to the measured numbers of particular types of inner nodes - see Section 3 and the average cost of their test.) It can be seen that theoretical estimates are quite close to experimental results. The original method needed more than three times as many edge tests. Table 4 shows how much this improvement is mirrored in running times for location and for the whole incremental insertion algorithm. Running times for location are for higher $n$ 2.56-2.85 times better than before; the whole running time is then $59-70 \%$ faster than before. 
Table 3. Number of tested edges per one point in the original DAG tests, in the proposed modification (New) and their rate for uniformly distributed data in $E^{2}$

\begin{tabular}{|c|c|c|c|c|c|c|c|}
\hline$n$ & 1,000 & 5,000 & 10,000 & 50,000 & 100,000 & 500,000 & $1,000,000$ \\
\hline Original & 86.23 & 113.95 & 130.008 & 156.20 & 163.88 & 194.25 & 204.68 \\
\hline New & 29.40 & 37.23 & 41.57 & 48.97 & 51.64 & 59.80 & 62.93 \\
\hline Orig./New - exper. & 2.93 & 3.06 & 3.13 & 3.19 & 3.17 & 3.25 & 3.25 \\
\hline Orig./New - theoret. & 2.81 & 2.81 & 2.81 & 2.81 & 2.81 & 2.81 & 2.80 \\
\hline
\end{tabular}

Table 4. Rate of running time for location and for the whole incremental insertion in $E^{2}$

\begin{tabular}{cccccccc}
\hline$n$ & 1,000 & 5,000 & 10,000 & 50,000 & 100,000 & 500,000 & $1,000,000$ \\
\hline Location; Orig./New & 9.75 & 4.76 & 2.56 & 2.85 & 2.71 & 2.57 & 2.61 \\
All; Orig./New & 1.70 & 1.63 & 1.59 & 1.68 & 1.66 & 1.69 & 1.70 \\
\hline
\end{tabular}

Tables 5 and 6 give results for $E^{3}$. Theoretical and practical results are in correspondence. Again, the original method needed more than three times as many face tests, however, location times are only 1.47-2.10 times better because some work needs to be spent to identify which face of the subdivided or swapped tetrahedron should be kept for further test and which not (in $E^{2}$ it is much easier to decide). The whole computation is improved only by $6-14 \%$ because in $E^{3}$, location is not so prevailing part of computation, taking up to $30-40 \%$ of the whole running time in comparison with $55-60 \%$ in $E^{2}$.

Table 5. Number of tested faces per one point in the original DAG tests, in the proposed modification (New) and their rate for uniformly distributed data in $E^{3}$

\begin{tabular}{|c|c|c|c|c|c|c|c|}
\hline$r$ & 1,000 & 5,000 & 10,000 & 50,000 & 100,000 & 200,000 & 240,000 \\
\hline Original & 155.82 & 220.64 & 242.23 & 303.00 & 334.60 & 357.88 & 361.27 \\
\hline New & 52.43 & 71.13 & 77.37 & 94.70 & 103.51 & 109.68 & 110.91 \\
\hline Orig./New - exper. & 2.97 & 3.10 & 3.13 & 3.20 & 3.23 & 3.26 & 3.26 \\
\hline Orig./New - theoret. & 3.30 & 3.31 & 3.31 & 3.31 & 3.31 & 3.31 & 3.31 \\
\hline
\end{tabular}

Table 6. Rate of running time for location and for the whole incremental insertion in $E^{3}$

\begin{tabular}{cccccccc}
\hline$n$ & 1,000 & 5,000 & 10,000 & 50,000 & 100,000 & 200,000 & 240,000 \\
\hline Location; Orig./New & 1.88 & 1.47 & 2.10 & 1.90 & 1.80 & 1.78 & 1.81 \\
All; Orig./New & 1.06 & 1.09 & 1.10 & 1.12 & 1.13 & 1.14 & 1.14 \\
\hline
\end{tabular}

\section{Conclusion}

We proposed a simple modification of DAG-based location for Delaunay incremental insertion in $E^{2}$ and $E^{3}$. The proposed method reduces about two thirds of tests, thus bringing a speedup of location especially in $E^{2}$. As only part of geometrical information about former simplices is needed, memory savings can be also achieved. 


\section{References}

1. de Berg M., van Kreveld M., Overmars M., Schwarzkopf O.: Computational Geometry. Algorithms and Applications, Springer-Verlag Berlin Heidelberg (1997)

2. Cignoni P., Montani C., Scopigno R.: DeWall: a Fast Divide and Conquer Delaunay Triangulation Algorithm in $E^{\mathrm{d}}$, Computer-Aided Design, Vol. 30, No.5 (1998) 333-341

3. Devillers O.: Improved Incremental Randomized Delaunay Triangulation, ACM Symposium on Computational Geometry, Minneapolis, USA (1998) 106-115

4. Devillers O., Pion S., Teillaud M.: Walking in a Triangulation, SCG'01 proceedings, Medford, Massachusets, USA (2001) 106-114

5. Erickson J.: Dense Point Sets Have Sparse Delaunay Triangulations or “... But Not Too Nasty", To appear in Proceedings of the 13th Annual ACM-SIAM Symposium on Discrete Algorithms (2002)

6. Erickson J.: Nice Point Sets Can Have Nasty Delaunay Triangulations, Proceedings of the 17th Annual ACM Symposium on Computational Geometry, (2001) 96-105. Invited to the special issue of Discrete \& Computational Geometry devoted to the symposium

7. Facello M.A.: Implementation of a Randomized Algorithm for Delaunay and Regular Triangulations in Three Dimensions, Computer Aided Geometric Design (1995) 349-370

8. Guibas L. J., Stolfi J.: Primitives for the Manipulation of General Subdivisions and the Computation of Voronoi Diagrams, ACM Transactions on Graphics, Vol. 4, No.2 (1985) $75-123$

9. Guibas L. J., Knuth D. E., Sharir M.: Randomized Incremental Construction of Delaunay and Voronoi Diagrams, Algorithmica, Vol. 7 (1992) 381-413

10.Joe B.: Three-dimensional Triangulations from Local Transformations, SIAM J. Sci. Stat. Comput. 10 (1989) 718-741

11.Joe B.: Construction of Three Dimensional Triangulations Using Local Transformations, Computer Aided Geometric Design 8 (1989) 123-142

12.Kolingerová I., Žalik B.: Improvements to Randomized Incremental Delaunay Insertion, sumitted to Computers and Graphics (2001)

13.Mulmuley K.: Randomized Multidimensional Search Trees: Dynamic Sampling. In: Proc. $7^{\text {th }}$ Annual ACM Sympos. Comput. Geom. (1991) 121-131

14.Mücke E. P., I. Saias I., Zhu B.: Fast Randomized Point Location Without Preprocessing in Two- and Three-dimensional Delaunay Triangulations, CompGeom'96, Philadelphia (1996) 274-283

15.Ohya T., Iri M., Murota K.: Improvements of the Incremental Method for the Voronoi Diagram with Computational Comparison of Various Algorithms, Journal of Operations Research Society of Japan, 27 (1984) 306-337

16.Sloan S. W.: A Fast Algorithm for Constructing Delaunay Triangulations in the Plane, Adv. Eng. Software, Vol. 9, No.1 (1987) 34-55

17.Su P., Drysdale R.L.S.: A Comparison of Sequential Delaunay Triangulation Algorithms, In: Proc. of the 11-th Annual Symposium on Computational Geometry (1995) 61-70, ACM

18.Vigo M.: An Improved Incremental Algorithm for Constructing Restricted Delaunay Triangulations, Computers \& Graphics 21 (1997) 215-223

19.Vigo M., Pla N.: Computing Directional Constrained Delaunay Triangulations, Computers \& Graphics 24 (2000) 181-190

20.Žalik B., Kolingerová I.: An Incremental Construction Algorithm for Delaunay Triangulation Based on Two-level Uniform Subdivision, submitted to International Journal of Geographical Information Science (2001) 